Nordic Machine Intelligence, MedAI 2021

https://doi.org/10.5617/nmi.9120

\title{
T-MIS: Transparency Adaptation in Medical Image Segmentation
}

\author{
Divij Singh ${ }^{1}$, Ayush Somani ${ }^{2,3}$, Alexander Horsch², Dilip K. Prasad ${ }^{2}$ \\ 1. Department of Computer Science, Indian Institute of Technology (BHU), Varanasi, India \\ 2. Department of Computer Science, UiT The Arctic University of Norway, Troms $\varnothing$, Norway \\ 3. E-mail any correspondence to: ayush.somani@uit.no
}

\begin{abstract}
We often locate ourselves in a trade-off situation between the model's prediction and understanding why the predictive modeling made such a prediction. This high-risk medical segmentation task is no different where we try to interpret what the model has learned from the image features irrespective of its high accuracy. We propose image-specific fine-tuning to make a deep learning model adaptive to specific medical imaging tasks. Experimental results reveal that: a) proposed model is more robust to segment previously unseen objects (negative test dataset) than state-of-the-art CNNs; b) image-specific fine-tuning with the proposed heuristics enhances segmentation accuracy significantly; and c) our model leads to accurate results with fewer user interactions and less user time than conventional interactive segmentation methods. The model successfully classified 'no polyp' or 'no instruments' in the image irrespective of the absence of polyp or instruments features in training samples from Kvasir-SEG and Kvasir-Instrument datasets.
\end{abstract}

Keywords: Image segmentation; transparency; medical imaging; image processing; deep learning

\section{Introduction}

Segmentation is considered among the most critical task in image analysis has gained pace with the advancement of accurate segmentation techniques. Fundamental segmentation decisions form the basis for image analysis like feature measurement, object representation, and description to high-level tasks like object classification. Manual interactions are subjected to improve accuracy by incorporating prior knowledge suffers from imperfection due to decision fatigue and tedious, time-consuming process, as well as the measures of training needed by operators [1]. These methods are also prone to reliability issues. Nevertheless, with increasing medical image modalities and an unmanageable quantity of medical images, the certainty of human intervention is questionable. However, automated segmentation techniques require some interaction for specifying few initial parameters, whose values can significantly impact performance [2]. The trade-off between manual interaction and performance is an essential factor in any segmentation application.

In medical science, researchers have identified the impact of segmentation and precision for diagnosis and treatment compared to mere classification and object detection with medical images [3]. Numerous methods that delineate a structure or an image include classical and pixel segmentation methods. Pixel segmentation like clustering, Markov Random Field, and deep learning approaches [4] is often the desired goal over classical segmentation like region-based, edge-based and thresholdbased segmentation [5] when disconnected regions with the same label class require identification. Several techniques are available specific to diagnosis application, imaging modality and area of interest in the body [6]. Simultaneously, validation experiments are necessary to add transparency to the model's performance. This transparency is helpful to the medical community as it defines the specific region of interest and provides some essential diagnostic information. The most straightforward approach to validation is to compare the automated segmentation with manually scored segmentation [7]. Besides the shortcomings of manual operation described above, this strategy does not promise a definite truth model because an operator's performance can also be flawed.

In this paper, colonoscopies is a reasonable use case for medical image segmentation and transparency tasks as it contains a large variety of findings that may go unnoticed. We propose a novel deep learningbased efficient segmentation technique along with the interpretation of the model's transparency performance on the polyp and instrument datasets [8,9] provided by the NORA MedAl challenge [10]. 


\section{Materials and methods}

Data Augmentations: The dataset followed a heuristic approach of augmentation that involves horizontal and vertical flipping of the image of $400 \times 400$-pixel size paired with increase/decrease brightness of value 40 . The algorithm has improved the task accuracy supported by the ablation study of the model and the basis that the datasets includes images with underexposed and overexposed image composition.

Training: Our method uses hybrid optimization techniques in artificial neural network using a DeeplabV3plus + ResNet101 as the backbone of data training to meet specific gastrointestinal image segmentation challenges. The model was trained on Kvasir-SEG [8] dataset using a 5-fold cross-validation technique of 800 training and 200 testing datasets. The training on the Kvasir-Instrument dataset [8] was performed only once pertaining to the dataset size. We use a CrossEntropyLoss with Adam Optimizer, a learning rate of 0.0001 , and training for 15 epochs. The batch size of 12 performed optimally compared to the standard size of 16 .

Transparency: Images are selected at random to preserve the generality of the model's interpretation. It is further evaluated with the base ground truth to prevent poor alignment correlating the explanation of the system's goal and the recipient's intention, i.e., understanding the prediction of conduct. An intuitive approach for visualizing actual differences in the segmentation, mainly at the boundary region of the positive class based on prior experience. The positive and learned features are extracted from the input image with the GT label mask and the generated mask. The feature attention and contour box plot comparing key model learned differences are represented in Figure 1 .

\section{Results}

Table 1 shows the segmentation performance of both polyp detection and instrument detection is achieved with an acceptable F1-score of $85 \%$ and $94.5 \%$ on the test datasets. For visualization, images are taken from the sample at random, representing the likeness in explanation for the interpretability strategies in action [11]. The lower right-most image pair in Figure 1, represents is the absence of instruments or polyp segmented truly is only present in the testing data.

\begin{tabular}{|c|c|c|c|c|c|c|}
\hline & P. Acc. & loU & Dice & Precision & Recall & F1 Score \\
\hline Dataset & \multicolumn{6}{|c|}{ Validation Metric Evaluation } \\
\hline SEG. [8] & 0.972 & 0.906 & 0.944 & 0.984 & 0.977 & 0.978 \\
\hline Instr. [9] & 0.992 & 0.952 & 0.973 & 0.994 & 0.998 & 0.997 \\
\hline Dataset & \multicolumn{6}{|c|}{ Test Metric Evaluation } \\
\hline SEG. [8] & 0.973 & 0.802 & 0.853 & 0.882 & 0.860 & 0.853 \\
\hline Instr. [9] & 0.991 & 0.908 & 0.945 & 0.923 & 0.974 & 0.945 \\
\hline
\end{tabular}

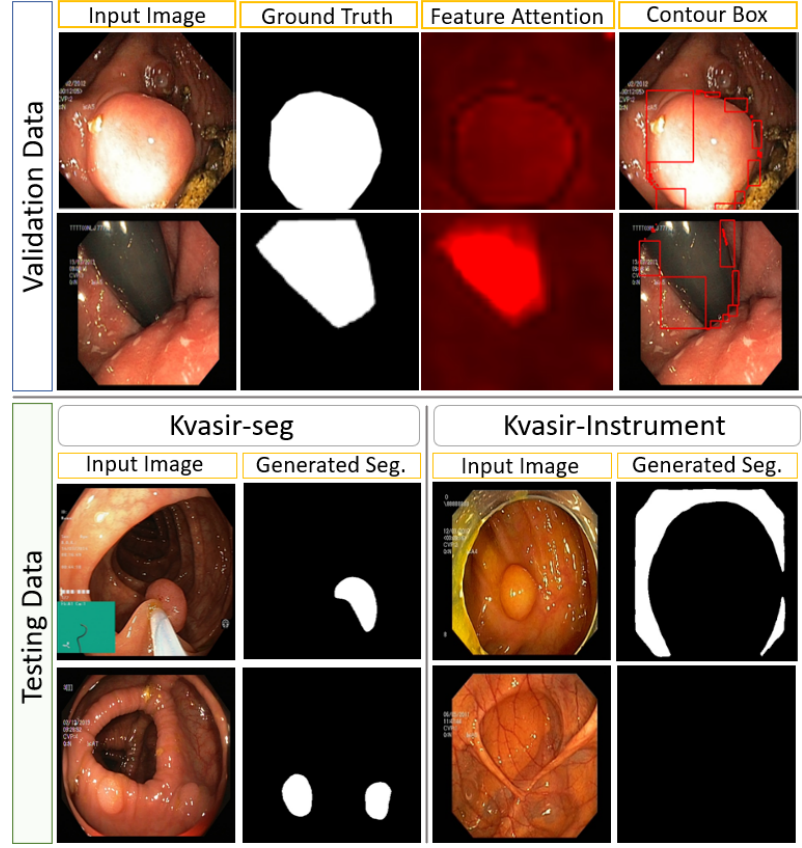

Figure 1: Qualitative results with transparency attributes for both Kvasir-seg [8] and Kvasir-Instrument [9] datasets.

\section{Discussion}

Several approaches in recent literature on medical image segmentation are used to solve different segmentation problems. We address the challenge using our hybrid deep learning-based model. The feature attention map in a single red channel in Figure 1 with density around the underlying boundary condition gives a good understanding of the model's learning and attention during training and validation. The thresholded feature differences and the model's attention on the pixel-wise learning behavior for the dataset are somewhat convincing. Still, it lacks the structure of interpretation needed in the high-risk medical domain. It is solved here by outlining the potential regions where human intervention, especially medical practitioners, can see the bounding area perceived as a challenge in learning. This can further assist annotate the data to increase the performance in the boundary states. The sureness in the health field is necessary, but even a diminutive visual interpretation can reveal a rich story incontrast to just statistical figures. In Figure 1, the explicit representation for both kinds of datasets helps even a nonmedical background conceive and interpret the efficacy of the automatic segmentation task. In the future, it will be interesting to improve the segmentation performance further and get more medically relevant explainability.

\section{Acknowledgement}

We acknowledge the funding support from the UiT Tematisk Project (Cristin no. 2061348) and Research Council of Norway INTPART grant no. 309802.

Table 1: Standard evaluation metrics for the tasks. 


\section{References}

1. Pham DL, $X u C$, and Prince JL. Current methods in medical image segmentation. Annual review of biomedical engineering 2000; 2:315-37

2. Davenport J, Bezdek J, and Hathaway R. Parameter estimation for finite mixture distributions. Computers \& mathematics with applications 1988; 15:819-28

3. Norouzi A, Rahim MSM, Altameem A, Saba T, Rad AE, Rehman $A$, and Uddin M. Medical image segmentation methods, algorithms, and applications. IETE Technical Review 2014; 31:199-213

4. Patil DD and Deore SG. Medical image segmentation: a review. International Journal of Computer Science and Mobile Computing 2013; 2:22-7

5. Michael E, Ma H, Li H, Kulwa F, and Li J. Breast Cancer Segmentation Methods: Current Status and Future Potentials. BioMed Research International 2021; 2021

6. Sharma N and Aggarwal LM. Automated medical image segmentation techniques. Journal of medical physics/Association of Medical Physicists of India 2010; 35:3

7. Wust P, Gellermann J, Beier J, Wegner S, Tilly W, Tröger J, Stalling D, Oswald H, Hege HC, Deuflhard P, et al. Evaluation of segmentation algorithms for generation of patient models in radiofrequency hyperthermia. Physics in Medicine \& Biology 1998; 43:3295

8. Jha D, Smedsrud PH, Riegler MA, Halvorsen P, Lange $T$ de, Johansen D, and Johansen HD. Kvasir-seg: $A$ segmented polyp dataset. International Conference on Multimedia Modeling. Springer. 2020 :451-62

9. Jha D, Ali S, Emanuelsen K, Hicks SA, Thambawita V, Garcia-Ceja E, Riegler MA, Lange T de, Schmidt PT, Johansen HD, et al. Kvasir-instrument: Diagnostic and therapeutic tool segmentation dataset in gastrointestinal endoscopy. International Conference on Multimedia Modeling. Springer. $2021: 218-29$

10. Hicks $S$, Jha D, Thambawita $\bigvee$, Riegler M, Halvorsen $P$, Singstad B, Gaur S, Pettersen K, Goodwin M, Parasa S, and Lange $T$ de. MedAl: Transparency in Medical Image Segmentation. Nordic Machine Intelligence 2021

11. Weller A. Transparency: motivations and challenges. Explainable Al: Interpreting, Explaining and Visualizing Deep Learning. Springer, $2019: 23-40$

12. Wang G, Li W, Zuluaga MA, Pratt R, Patel PA, Aertsen M, Doel $T$, David AL, Deprest J, Ourselin S, and Vercauteren $T$. Interactive Medical Image Segmentation Using Deep Learning With Image-Specific Fine Tuning. IEEE Transactions on Medical Imaging 2018; 37:156273. DOI: $10.1109 /$ TMI.2018.2791721

13. Srivastava A, Jha D, Chanda S, Pal U, Johansen HD, Johansen D, Riegler MA, Ali S, and Halvorsen P. MSRF-Net: A Multi-Scale Residual Fusion Network for Biomedical Image Segmentation. arXiv preprint arXiv:2105.07451 2021
14. Borgli $H$, Thambawita $\bigvee$, Smedsrud PH, Hicks S, Jha D, Eskeland SL, Randel KR, Pogorelov K, Lux M, Nguyen DTD, et al. HyperKvasir, a comprehensive multi-class image and video dataset for gastrointestinal endoscopy. Scientific data 2020; 7:1-14 